\title{
Determinación de hierro en aceitunas negras mediante extracción con ácido tricloroacético
}

\author{
Por P. García García, M. Brenes Balbuena, F. Baraud ${ }^{\star \star}$ y A. Garrido Fernández* \\ U.E.I. de Biotecnología de Alimentos. Instituto de la Grasa y sus \\ Derivados. Aptdo. 1048 Sevilla. España. \\ ** Becario COMETT.
}

\section{RESUMEN}

Determinación de hierro en aceitunas negras mediante extracción con ácido tricloroacético.

En el trabajo se estudia la extracción del hierro con ácido tricloroacético, en sustitución de la mineralización, en el transcurso del análisis de dicho catión con o-fenantrolina en las aceitunas tipo negras. Los resultados obtenidos con la nueva metodología indican la ausencia de errores sistemáticos y proporcionales, dando valores estadísticamente iguales y con la misma precisión que con la mineralización. Por tanto, se recomienda su uso para el control de este metal en dicho producto, especialmente en la industria por su mayor rapidez y economía.

PALABRAS - CLAVE: Aceituna negra por oxidación - Acido tricloroacético - Hierro (determinación).

SUMMARY

Iron determination in ripe olives by trichloroacetic acid extraction.

The paper studies the use of the iron extraction instead of the mineralization, for the iron analysis in ripe olives with o-phenantroline. Results did not show systematic and proportional errors. They were statistically equal and had the same precision. Consequently, the procedure is suitable for the iron control in ripe olives, especially in the industry laboratories, as it is quicker and cheaper than the mineralization.

KEY-WORDS: Iron (determination) - Ripe olive - Trichloroacetic acid.

\section{INTRODUCCION}

La oxidación en medio alcalino de los compuestos fenólicos presentes en la pulpa de los frutos origina el color negro característico de las denominadas aceitunas tipo negras (Brenes et al, 1992). Asimismo, es una práctica habitual en la industria adicionar sales de hierro durante la fase de aireación y envasado de las mismas con objeto de fijar dicho color negro (Fernández et al, 1985). Para ello, los únicos compuestos autorizados internacionalmente hasta ahora son el lactato y el gluconato ferroso, siendo este último el más utilizado. Las Normas Internacionales COI/CODEX permiten hasta $0,15 \mathrm{~g}$ de hierro total por $\mathrm{Kg}$ de aceitunas, aunque en algunos países la legislación es más estricta.

Ya que el gluconato ferroso suele ser la sal más empleada, se han desarrollado diversos métodos de determinación del anión para estimar la cantidad de aditivo usado (Guzmán et al, 1980; Hirokado et al, 1985). No obstante, operando de esta forma se obvia el contenido natural de hierro en la pulpa de los frutos que a veces puede ser considerable. Por ello, el método de análisis más empleado para cuantificar el contenido total de este metal en las aceitunas es el desarrollado por Albi y Garrido (1975), que consiste en la mineralización de la muestra, la solubilización del catión con $\mathrm{HCl}$ y la valoración del contenido del mismo mediante la reacción colorimétrica del ion ferroso con la ofenantrolina. Este procedimiento, no es fácil de aplicar en la industria debido, en gran medida, a la lentitud de la fase de mineralización y la falta de equipos adecuados en los laboratorios de dichas instalaciones. Por ello, diversos autores han propuesto el empleo de agentes quelantes para la separación de los elementos minerales de los frutos, como una alternativa a la mineralización. Entre estas sustancias destaca el ácido tricloroacético (TCA), el cual permite la extracción de gran parte de los cationes presentes en aquéllos (Leggett y Westerman, 1973) (Salazar y Young, 1984).

El objetivo del presente trabajo consiste en desarrollar un método de análisis del contenido en hierro en la pulpa de aceitunas y en los líquidos de gobierno que evite la fase de mineralización y que, por su simplicidad, pueda emplearse de forma rutinaria en la industria.

\section{PARTE EXPERIMENTAL}

\subsection{Aceitunas}

En todas las experiencias se emplearon frutos de la variedad Hojiblanca conservados durante un año y oxidados en medio alcalino según el método habitual (Fernández et al, 1985).

\subsection{Método de extracción con TCA}

\section{MATERIALES Y REACTIVOS:}

- Tubos de ensayos de 40 ml de capacidad con tapón. - Embudos. 
- Papel de filtro: Albet $n^{0} 305$ o equivalente.

- Acido tricloroacético: solución al $4 \%$ en agua desionizada .

- Carbón activo: tipo GA (Industrias Kern, Barcelona).

\section{MODO OPERATIVO:}

Al igual que en la mineralización, previamente es necesaria la trituración de la pulpa, añadiendo para facilitarla la misma cantidad de fruto que de agua desionizada. De esta mezcla se toman $4,0 \mathrm{~g}$, exactamente pesados, que se colocan en un tubo de ensayo al que se añaden $30 \mathrm{ml}$ de la solución de TCA y 0,3 g de carbón activo. Una vez cerrado se agita enérgicamente de vez en cuando durante media hora y se filtra a través de papel. Este filtrado constituye el extracto mineral para realizar la reacción colorimétrica. Para los líquidos de gobierno del envasado se toman directamente $5 \mathrm{ml}$ de salmuera, se añaden $20 \mathrm{ml}$ de la solución de TCA y $0,5 \mathrm{~g}$ de carbón activo, operándose de igual forma que para la pulpa.

En ambos casos, para la reacción colorimétrica se utilizan $5 \mathrm{ml}$ del extracto. De esta forma, la cantidad de hierro en el volumen final queda comprendida dentro de los valores de la recta patrón (Albi y Garrido, 1975). Para los cálculos se considera que los $4 \mathrm{~g}$ de mezcla ocupan un volumen de $3 \mathrm{ml}$.

Como en la mineralización, es necesario realizar un ensayo en blanco, tomando las mismas cantidades de reactivos y, en vez de la muestra, agua desionizada.

\subsection{Diseño de las experiencias}

En primer lugar se realiza el estudio espectrofotométrico de la posible interferencia del color de la solución de extracción con TCA en la absorbancia a $508 \mathrm{~nm}$ y la forma de eliminarlo.

La comparación del método de extracción con TCA y el de mineralización, se efectúa, tanto para la pulpa como para los líquidos de gobierno, analizando por ambos procedimientos triplicados de diferentes muestras.

El estudio de la regresión correspondiente permite conocer si los valores de la pendiente y la ordenada en el origen incluyen respectivamente el 1 y 0 , lo que indicará que las dos extracciones dan lugar a los mismos resultados. Por otra parte, la relación de las varianzas, servirá mediante la prueba de F, para establecer si los dos procedimientos son igualmente precisos (Massart, 1978).

\section{RESULTADOS}

En la Figura 1 se recoge el espectro de absorción del complejo $\mathrm{Fe}^{++}$-o-fenantrolina del extracto mineral de pulpa de aceituna obtenido con TCA. Como puede observarse, el máximo esperado a $508 \mathrm{~nm}$ no está definido, debido a la absorción de la solución del extracto mineral (con la misma dilución) en esta zona del espectro (curva inferior), ya que además de los cationes, se disuelven también en la solución de extracción otras sustancias orgánicas responsables de la coloración de las aceitunas.

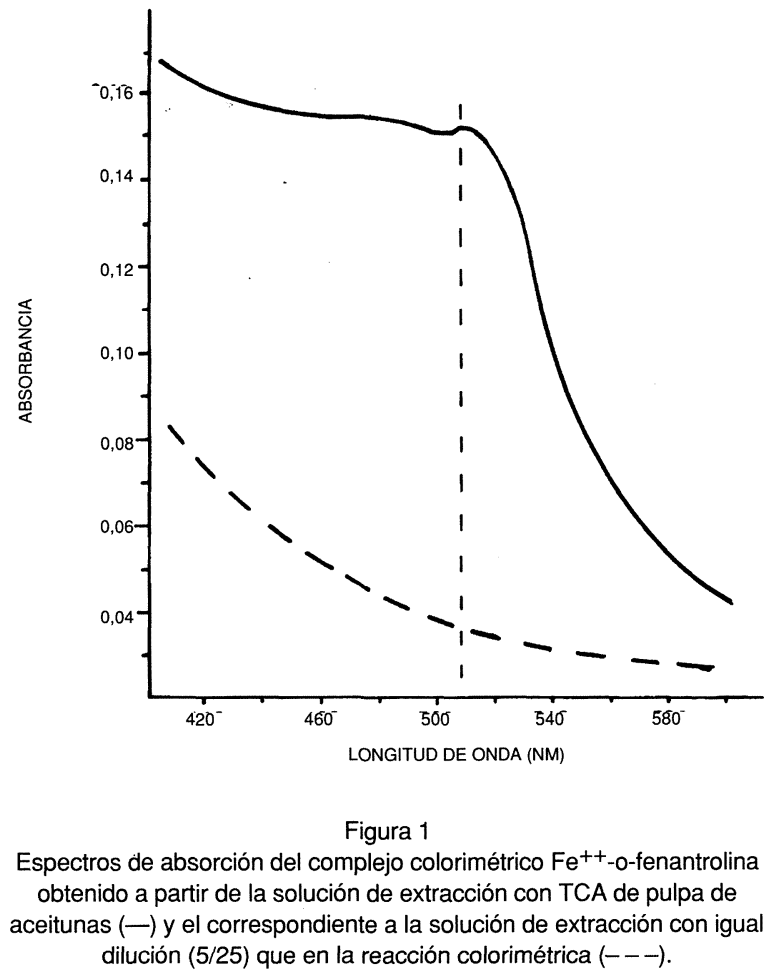

Por tanto, la absorbancia medida a $508 \mathrm{~nm}$ no corresponde únicamente a la propia de la reacción colorimétrica (la relacionada con la concentración del complejo $\mathrm{Fe}^{++}-0-$ fenantrolina), sino a la suma de ésta y la debida a la solución de extracción. En consecuencia, el procedimiento introduce, si no se corrige, un error sistemático.

Exactamente lo mismo ocurre cuando se trabaja con los líquidos de gobierno.

Es, pues, absolutamente necesario en ambos casos eliminar la coloración de la solución de extracción antes de efectuar la reacción colorimétrica. Con este objetivo se han ensayado diferentes compuestos. El uso de polivinil-pirrolidona no produjo una buena decoloración incluso utilizando concentraciones de hasta $2 \mathrm{~g} / 2 \mathrm{~g}$ de pulpa. Sin embargo, el carbón activo, tipo GA (Industrias Kern, Barcelona), del que se había demostrado que retenía los fenoles polimerizados responsables del color en las salmueras de conservación de aceitunas verdes estilo sevillano (Brenes et al, 1988), sí da buenos resultados.

La cantidad de carbón activo necesario para evitar la interferencia se determinó analizando en los extractos de muestras de pulpa y líquido de gobierno, diluidos $5 / 25$, la absorbancia residual a $508 \mathrm{~nm}$, en función de los gramos de carbón añadido (Figura 2).

De acuerdo con los resultados, la cantidad necesaria para eliminar la interferencia debe ser de $0,3 \mathrm{~g}$ por cada 2 $\mathrm{g}$ de pulpa y $0,5 \mathrm{~g}$ por cada $5 \mathrm{ml}$ de líquido de gobierno.

En la Figura 3 se presentan los espectros de absorción 


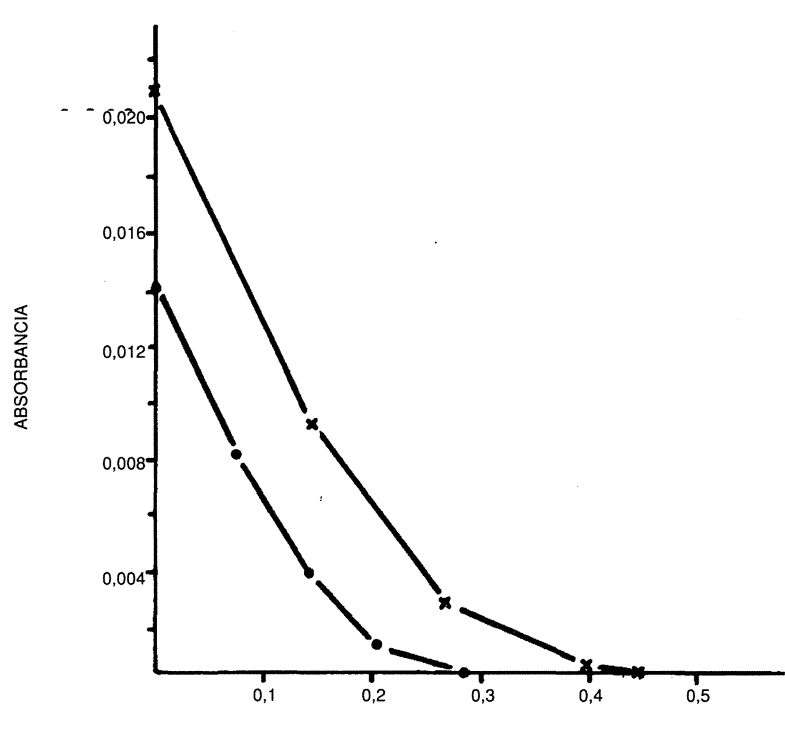

CARBON ACTIVO $(\mathrm{g})$

\section{Figura 2}

Absorbancia a $508 \mathrm{~nm}$ de la solución diluida (5/25) del extracto de TCA de la pulpa (.) y de los líquidos de gobierno ( $x$ ) de envasados de aceitunas tipo negras en función de la cantidad de carbón activo usado para la decoloración.

del complejo colorimétrico obtenido a partir de extractos de pulpa y líquido de gobierno tratados con las cantidades antes indicadas de carbón activo. En dichas gráficas está definido el máximo característico de absorbancia a $508 \mathrm{~nm}$ del complejo $\mathrm{Fe}^{++}-$-fenantrolina, en contraste con lo que ocurría cuando la reacción se efectuaba sin decoloración previa del extracto mineral (Figura 1), que no quedaba nítidamente definido.

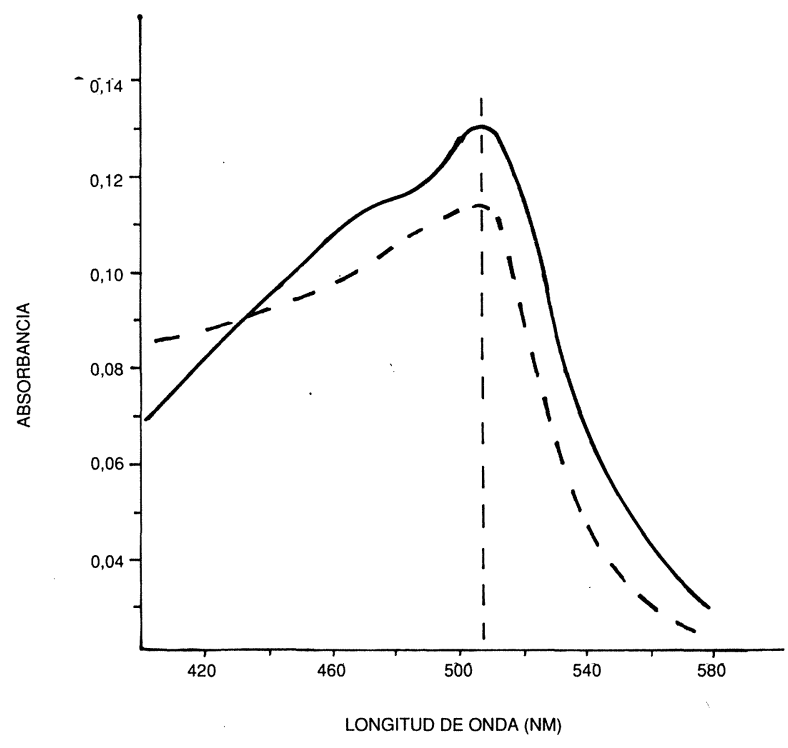

Figura 3

Espectros de absorción del complejo colorimétrico $\mathrm{Fe}^{++}$-o-fenantrolina obtenidos a partir de extractos de TCA de pulpa $(-)$ y de líquidos de gobierno $(--)$ de aceitunas tipo negras previamente decoloradas con carbón activo $(0,3 \mathrm{~g} / 2 \mathrm{~g}$ pulpa y $0,5 \mathrm{~g} / 5 \mathrm{ml}$ salmuera, respectivamente).
Por otra parte, en la Tabla I se recogen los resultados medios del contenido en hierro de pulpas y líquidos de gobierno en una serie de muestras, determinadas por el método de la o-fenantrolina, tanto por el procedimiento de mineralización por vía seca como por el de extracción con TCA y adición de carbón activo.

Tabla I

- Resultados de las determinaciones del contenido en hierro (ppm) en pulpa y líquido de gobierno de envasados de aceitunas tipo negras. Comparación de los dos métodos de preparación de la muestra ensayados: mineralización y extracción con TCA

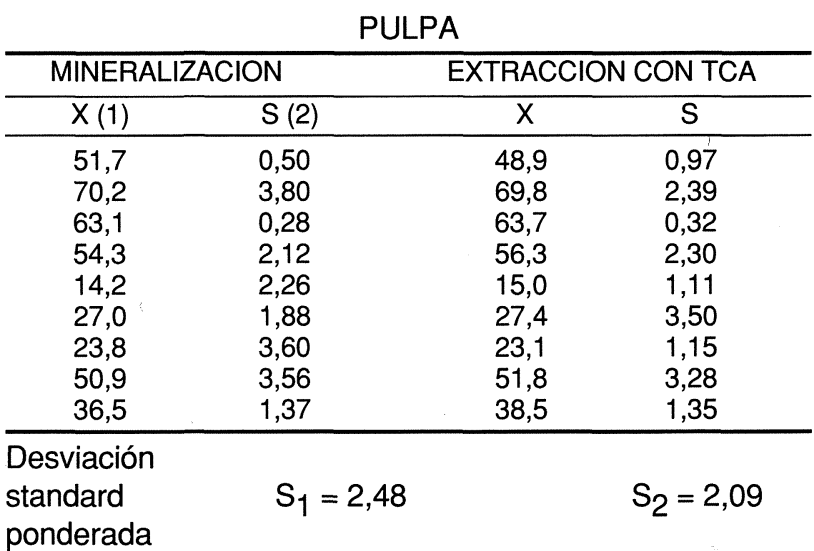

Comparación de las varianzas $F^{\star}=\frac{S_{1}{ }^{2}}{S_{2}{ }^{2}}=1,40$

$F(18,18,0.05)=2,22$

LIQUIDO DE GOBIERNO

\begin{tabular}{cccc}
\hline \multicolumn{2}{c}{ MINERALIZACION } & \multicolumn{2}{c}{ EXTRACCION CON TCA } \\
\hline $\mathrm{X}$ & $\mathrm{S}$ & $\mathrm{X}$ & $\mathrm{S}$ \\
\hline 20,1 & 0,51 & 20,0 & 1,20 \\
13,8 & 0,30 & 14,5 & 1,20 \\
18,1 & 0,92 & 19,0 & 0,52 \\
20,0 & 1,50 & 19,9 & 0,42 \\
14,7 & 1,75 & 14,7 & 0,26 \\
10,6 & 0,61 & 10,2 & 0,68 \\
19,4 & 0,46 & 19,0 & 1,34 \\
\hline Desviación & & & \\
standard & $\mathrm{S}_{3}=1,00$ & & $\mathrm{~S}_{4}=1,19$
\end{tabular}

ponderada
Comparación de las varianzas
$F(14,14,0.05)=2,51$

NOTAS: (1) Media de tres determinaciones; (2) Desviación standard. 
Los parámetros de la regresión obtenida al comparar los resultados de los dos métodos: mineralización $(x)$ y extracción con TCA (y), se recogen en la Tabla II. Como puede observarse las rectas, tanto para la pulpa como para los líquidos, tienen pendientes y ordenadas en el origen cuyos limites de confianza incluyen la unidad y cero, respectivamente. Ello significa que los valores obtenidos a partir de los dos sistemas de extracción son estadísticamente iguales y sin que aparezcan errores sistemáticos ni proporcionales.

En la Tabla I se recoge asimismo el error standard de las medias de los replicados y el combinado de cada método. La aplicación de la prueba de $\mathrm{F}$ pone de manifiesto que, tanto para la pulpa como para los líquidos, las varianzas obtenidas cuando se determina el contenido en hierro mediante la mineralización o la extracción con TCA, son estadísticamente iguales. Por tanto, los dos métodos tienen la misma precisión.

Tabla II

Estimación de los parámetros de la recta de regresión $(y=a+b x)$, obtenida al comparar los resultados de la determinación de hierro por los métodos de mineralización $(x)$ y extracción con TCA $(y)$ de pulpa $y$ líquido de gobierno de envasados de aceitunas tipo negras

\begin{tabular}{|c|c|c|}
\hline & PULPA & $\begin{array}{l}\text { LIQUIDO DE } \\
\text { GOBIERNO }\end{array}$ \\
\hline COEFICIENTE DE & & \\
\hline $\begin{array}{l}\text { CORRELACION (r): } \\
\text { ORDENADA EN EL ORIGEN (a) }\end{array}$ & 0,997 & 0,990 \\
\hline Valor: & 0,669 & 0,090 \\
\hline $\begin{array}{l}\text { Límites de confianza: } \\
\text { PENDIENTE (b) }\end{array}$ & $3,80>a>-2,50$ & $2,70>a>-2,50$ \\
\hline Valor: & 0,992 & 0,999 \\
\hline Limites de confianza: & $1,06>b>0,92$ & $1,09>b>0,91$ \\
\hline
\end{tabular}

\section{CONCLUSION}

Según todo lo expuesto anteriormente, la extracción con TCA puede sustituir ventajosamente por su mayor facilidad y rapidez a la mineralización en la preparación de las muestras para la determinación del contenido en hierro en la pulpa y líquidos de gobierno de las aceitunas tipo negras.
La determinación mediante la reacción colorimétrica con la o-fenantrolina se debe llevar a cabo con la solución del extracto mineral decolorado para evitar interferencias. Ello, se consigue mediante la adición de carbón activo tipo GA (Industrias Kern, Barcelona) a razón de $0,3 \mathrm{~g}$ por cada $2 \mathrm{~g}$ de pulpa y de $0,5 \mathrm{~g}$ por cada $5 \mathrm{ml}$ de líquido de gobierno que se tomen de muestra.

Los resultados obtenidos con ambos procedimientos de preparación de la muestra son estadísticamente iguales, así como sus precisiones, no apreciándose errores sistemáticos ni proporcionales.

\section{AGRADECIMIENTO}

A la CICYT por la financiación del proyecto Ali-91-1166CO3-01 y al programa COMETT de la CEE por la beca concedida al autor F. Baraud.

\section{BIBLIOGRAFIA}

Albi, M.A. y Garrido, A. (1975).- "Aceitunas negras por oxidación en medio alcalino. III. Estudio comparativo de métodos de determinación de hierro".- Grasas y Aceites 26,133-135.

Brenes, M. y Garrido, A. (1988).- "Regeneración de salmuera de aceitunas verdes estilo español con carbón activo y tierras decolorantes".- Grasas y Aceites 39, 96-101.

Brenes, M. García, P. and Garrido, A. (1992) - "Phenolic compounds related to the black color formed during the processing of ripe olives".- J. Agric. Food Chem. 40, 1192-1196.

Fernández, M .J.; Castro, R.; Garrido, A.; González, F.; Nosti, M .; Heredia, A.; Mínguez, M.I.; Rejano, L.; Durán, M.C.; Sánchez, F.; García, P. y Castro, A. (1985).-"Biotecnología de la aceituna de mesa".-Instituto de la Grasa y sus Derivados. CSIC. Sevilla.

Guzmán, M.; Bautista, F.J. y Garrido, A. (1980).- "Determinación de gluconato ferroso en aceituna de mesa".- Anal. Bromatol. 32, 299-302.

Hirokado, M.; Nakajima, K; Takahashi, I.; Rematsu, K.; Endo, F. and Kazama, M. (1985).-"Method for quantitative determination of ferrous gluconate in table olive".- Annual Report of Tokyo Metropolitan Research Laboratory of Public Health. 36, 219-233.

Leggett, G.E. and Westerman, D. (1973).-"Determination of mineral elements in plant tissues using trichloroacetic acid extraction". -J . Agric. Food Chem. 21, 65-69.

Massart, D.L. (1978).-"Evaluation and optimization of laboratory methods and analytical procedures".- Ed. Elsevier, Amsterdam.

Salazar, A.J. and Young, C.T (1984).- "An automated methylthynol the method for calcium determination in plants".- J. Food Sci. 49, 209-211.

(Recibido: Noviembre 1992) 